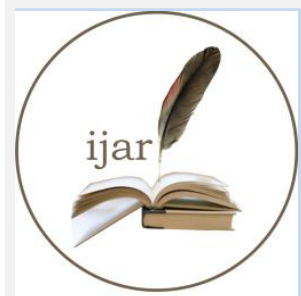

ISSN NO. 2320-5407

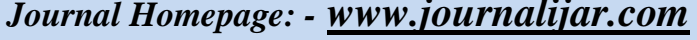 INTERNATIONAL JOURNAL OF ADVANCED RESEARCH (IJAR)}

Article DOI: $10.21474 / \mathrm{IJAR01/2202}$

DOI URL: http://dx.doi.org/10.21474/IJAR01/2202
INTERNATIONAL JOURNAL OF ADVANCED RESEARCH (JJAR)

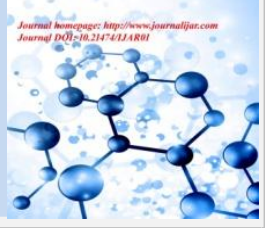

RESEARCH ARTICLE

\title{
A CLASS OF INTEGRAL TRANSFORM.
}

Sachin Sharma and A.K. Ronghe.

Department of Pure and Applied Mathematics S.S.L. Jain P.G. College, Vidisha (M.P.)-464001 (India)

\section{Manuscript Info}

\section{Manuscript History}

Received: 28 September 2016

Final Accepted: 30 October 2016

Published: November 2016

Key words:-

Hypergeometric function, Fox's H-

function I-function of two variables,

Two dimensional I-function transform,

etc.

\section{Abstract}

In this research paper, we discuss new class of integral transform whose kernel involved a product of exponential function and Ifunction of two variables defined by K. Shantha Kumari, Vasudevan Nambisan and A.K. Rathie [2]. In the last particular cases of two dimensional integral transform are also discussed.

\section{Introduction:-}

The double Mellin Barnes type contour integral occurring in this paper will be defined by [2] K. Shantha Kumari, T.M. Vasudevan Nambisan and Rathie A.K. and represented in the following manner.

$$
\begin{aligned}
& \mathrm{I}_{\mathrm{p}_{1}, \mathrm{q}_{1} ; \mathrm{N}_{2}}^{0, \mathrm{n}_{1} ; \mathrm{N}_{1}}\left[\begin{array}{l|l}
\mathrm{z}_{1} & ((\mathrm{P})),((\mathrm{Q})),((\mathrm{R})) \\
\mathrm{z}_{2} & ((\mathrm{~S})),((\mathrm{T})),((\mathrm{U}))
\end{array}\right] \\
& =\frac{1}{-4 \pi^{2}} \int_{\mathcal{L}_{\mathrm{s}}} \int_{\mathcal{L}_{\mathrm{t}}} \phi(\mathrm{S}, \mathrm{t}) \theta_{1}(\mathrm{~s}) \theta_{2}(\mathrm{t}) \mathrm{z}_{1}{ }^{\mathrm{s}} \mathrm{z}_{2}{ }^{\mathrm{t}} \mathrm{dsdt}
\end{aligned}
$$

Where $\phi(\mathrm{s}, \mathrm{t}), \theta_{1}(\mathrm{~s}), \theta_{2}(\mathrm{t})$ are given by

$$
\phi(s, t)=\frac{\prod_{j=1}^{n_{1}} \Gamma^{\xi_{j}}\left(1-a_{j}+\alpha_{j} s+A_{j} t\right)}{\prod_{j=n_{1}+1}^{p_{1}} \Gamma^{\xi_{j}}\left(a_{j}-\alpha_{j} s-A_{j} t\right) \prod_{j=1}^{q_{1}} \Gamma^{\eta_{j}}\left(1-b_{j}+\beta_{j} s+B_{j} t\right)},
$$

\section{Corresponding Author:- Sachin Sharma.}

Address:- Department of Pure and Applied Mathematics S.S.L. Jain P.G. College, Vidisha (M.P.)464001 (India). 


$$
\begin{aligned}
& \theta_{1}(\mathrm{~s})=\frac{\prod_{\mathrm{j}=1}^{\mathrm{n}_{2}} \Gamma^{U_{j}}\left(1-\mathrm{c}_{\mathrm{j}}+\mathrm{C}_{\mathrm{j}} s\right) \prod_{\mathrm{j}=1}^{\mathrm{m}_{2}} \Gamma^{V_{j}}\left(\mathrm{~d}_{\mathrm{j}}-\mathrm{D}_{\mathrm{j}} s\right)}{\prod_{\mathrm{j}=\mathrm{n}_{2}+1}^{\mathrm{p}_{2}} \Gamma^{U_{j}}\left(\mathrm{c}_{\mathrm{j}}-\mathrm{C}_{\mathrm{j}} s\right) \prod_{\mathrm{j}=\mathrm{m}_{2}+1}^{\mathrm{q}_{2}} \Gamma^{V_{j}}\left(1-\mathrm{d}_{\mathrm{j}}+\mathrm{D}_{\mathrm{j}} s\right)}, \\
& \theta_{2}(\mathrm{t})=\frac{\prod_{\mathrm{j}=1}^{\mathrm{n}_{3}} \Gamma^{P_{j}}\left(1-\mathrm{e}_{\mathrm{j}}+\mathrm{E}_{\mathrm{j}} t\right) \prod_{\mathrm{j}=1}^{\mathrm{m}_{3}} \Gamma^{Q_{j}}\left(\mathrm{f}_{\mathrm{j}}-\mathrm{F}_{\mathrm{j}} t\right)}{\prod_{\mathrm{j}=\mathrm{n}_{3}+1}^{\mathrm{q}_{3}} \Gamma^{P_{j}}\left(\mathrm{e}_{\mathrm{j}}-\mathrm{E}_{\mathrm{j}} t\right) \prod_{\mathrm{j}=\mathrm{m}_{3}+1} \Gamma^{Q_{j}}\left(1-\mathrm{f}_{\mathrm{j}}+\mathrm{F}_{\mathrm{j}} t\right)},
\end{aligned}
$$

Also:

- $z_{1} \neq 0, z_{2} \neq 0$;

* $i=\sqrt{-1}$;

* an empty product is interpreted as unity;

* $\mathcal{L}_{s}$ and $\mathcal{L}_{t}$ are suitable contours of Mellin-Barnes type. Moreover, the contour $\mathcal{L}_{s}$ is in the complex s-plane and runs from $\sigma_{1}-i \infty$ to $\sigma_{1}+i \infty,\left(\sigma_{1}\right.$ real $)$ so that all the singularities of $\quad \Gamma^{V_{j}}\left(d_{j}-D_{j} s\right)\left(j=1, \ldots \ldots, m_{2}\right)$ lies to the right of $\mathcal{L}_{s}$ and all the singularities of $\Gamma^{U_{j}}\left(1-c_{j}+C_{j} s\right)\left(j=1, \ldots \ldots, n_{2}\right)$, $\Gamma^{\xi_{j}}\left(1-a_{j}+\alpha_{j} s+A_{j} t\right)\left(j=1, \ldots \ldots, n_{1}\right)$ lie to the left of $\mathcal{L}_{s} ;$

* The contour $\mathcal{L}_{t}$ is in the complex t-plane and runs from $\sigma_{2}-i \infty$ to $\sigma_{2}+i \infty,\left(\sigma_{2}\right.$ real $)$ so that all the singularities of $\Gamma^{Q_{j}}\left(f_{j}-F_{j} t\right)\left(j=1, \ldots \ldots, m_{3}\right)$ lie to the right of $\mathcal{L}_{t}$ and all the singularities of $\Gamma^{P_{j}}\left(1-e_{j}+E_{j} t\right)\left(j=1, \ldots \ldots, n_{3}\right), \Gamma^{\xi_{j}}\left(1-a_{j}+\alpha_{j} s+A_{j} t\right)\left(j=1, \ldots \ldots, n_{1}\right)$ lie to the left of $\mathcal{L}_{t}$.

For the condition of existence and condition on the various parameters of I-function of two variables $I\left[Z_{1}, Z_{2}\right]$ we refer to $[2,5]$ in (1.1) and that follows, we use the following notations for the sake of brevity.

$\mathrm{N}_{1} \equiv m_{2}, n_{2}: m_{3}, n_{3} ; \quad \mathrm{N}_{2} \equiv p_{2}, q_{2}: p_{3}, q_{3} ;$

And sets of parameters are

$((P)) \equiv\left(a_{j}, \alpha_{j}, A_{j} ; \xi_{j}\right)_{1, p_{1}},((Q)) \equiv\left(c_{j}, C_{j} ; U_{j}\right)_{1, p_{2}},((R)) \equiv\left(e_{j}, E_{j} ; P_{j}\right)_{1, p_{3}}$

$((S)) \equiv\left(b_{j}, \beta_{j}, B_{j} ; \eta_{j}\right)_{1, q_{1}},((T)) \equiv\left(d_{j}, D_{j} ; V_{j}\right)_{1, q_{2}},((U)) \equiv\left(f_{j}, F_{j} ; Q_{j}\right)_{1, q_{3}}$

Following the results of Braaksma [1, p.378] and Rathie [2, 7, 10, 11], it can easily be shown that the function defined in (1.1) is analytic function of $Z_{1}$ and $Z_{2}$ if $\mathrm{R}<0$ and $\mathrm{S}<0$. Where

$$
\begin{aligned}
& \mathrm{R}=\sum_{j=1}^{p_{1}} \xi_{j} \alpha_{j}+\sum_{j=1}^{p_{2}} U_{j} C_{j}-\sum_{j=1}^{q_{2}} \eta_{j} \beta_{j}-\sum_{j=1}^{q_{2}} V_{j} D_{j}, \\
& \mathrm{~S}=\sum_{j=1}^{p_{1}} \xi_{j} A_{j}+\sum_{j=1}^{p_{3}} P_{j} E_{j}-\sum_{j=1}^{q_{1}} \eta_{j} B_{j}-\sum_{j=1}^{q_{3}} Q_{j} F_{j},
\end{aligned}
$$


And the integral (1.1) is convergent if,

$$
\Delta_{1}>0, \Delta_{2}>0,\left|\arg \left(z_{1}\right)\right|<\frac{1}{2} \Delta_{1} \pi,\left|\arg \left(z_{2}\right)\right|<\frac{1}{2} \Delta_{2} \pi,
$$

Where,

$$
\begin{aligned}
& \Delta_{1}=\left[\sum_{j=1}^{n_{1}} \xi_{j} \alpha_{j}-\sum_{j=n_{1}+1}^{p_{1}} \xi_{j} \alpha_{j}-\sum_{j=1}^{q_{1}} \eta_{j} \beta_{j}+\sum_{j=1}^{n_{2}} U_{j} C_{j}-\sum_{j=n_{2}+1}^{p_{2}} U_{j} C_{j}+\sum_{j=1}^{m_{2}} V_{j} D_{j}-\sum_{j=m_{2}+1}^{q_{2}} V_{j} D_{j}\right], \\
& \Delta_{2}=\left[\sum_{j=1}^{n_{1}} \xi_{j} A_{j}-\sum_{j=n_{1}+1}^{p_{1}} \xi_{j} A_{j}-\sum_{j=1}^{q_{1}} \eta_{j} B_{j}+\sum_{j=1}^{n_{3}} P_{j} E_{j}-\sum_{j=n_{3}+1}^{p_{3}} P_{j} E_{j}+\sum_{j=1}^{m_{3}} Q_{j} F_{j}-\sum_{j=m_{3}+1}^{q_{3}} Q_{j} F_{j}\right],
\end{aligned}
$$

Integral (1.1) is convergent absolutely if

$$
\Delta_{1} \geq 0, \Delta_{2} \geq 0,\left|\arg \left(z_{1}\right)\right|=\frac{1}{2} \Delta_{1} \pi,\left|\arg \left(z_{2}\right)\right|=\frac{1}{2} \Delta_{2} \pi
$$

\section{Transform for I-function of two variables with respect to 2-D:-}

In this section we shall define the 2-D integral transform of the function $f(x, y)$ whose kernel involving a product of the exponential function and I-function of two variables defined by K. Shantha Kumari, Nambisan Vasudevan and Rathie, A.K. [2], which will be defined as follows:

$$
\phi[f ; s, t]=\int_{0}^{\infty} \int_{0}^{\infty} e^{\frac{-s x}{a}} e^{\frac{-t y}{b}} \mathrm{I}_{\mathrm{p}_{1}, \mathrm{q}_{1} ; \mathrm{N}_{2}}^{0, \mathrm{n}_{1} ; \mathrm{N}_{1}}\left[\begin{array}{c|c|c|}
\alpha s x & ((\mathrm{P})),((\mathrm{Q})),((\mathrm{R})) \\
\beta t y & ((\mathrm{~S})),((\mathrm{T})),((\mathrm{U}))
\end{array}\right] f(x, y) d x d t
$$

Where, $0<a<\infty, 0<b<\infty, s, t \neq 0$ and $f(x, y)$ is real and complex valued function of two variables $x$ and $y$.

$((\mathrm{P})),((\mathrm{Q})),((\mathrm{R})),((\mathrm{S})),((\mathrm{T}))$ and $((\mathrm{U}))$ are sets of parameters given in the previous section and $a, b$ lies between $(0, \infty)$ such that the product $x^{\alpha} \cdot y^{\beta} \cdot f(x, y)$ is integrable over the finite region,

$$
D:\left(D_{1}, D_{2}\right): 0 \leq x \leq D_{1}, 0 \leq y \leq D_{2}, D_{1}>0, D_{2}>0 \text {, }
$$

Where

$$
\alpha=\min _{0 \leq j \leq m_{2}}\left[\operatorname{Re}\left(\frac{d_{j} V_{j}}{D_{j}}\right)\right], \quad \beta=\underset{0 \leq j \leq m_{3}}{\min }\left[\operatorname{Re}\left(\frac{f_{j} Q_{j}}{F_{j}}\right)\right],
$$

We shall denote the two dimensional I-function transform of the function $f(x, y)$ by

$$
\mathrm{I}_{\mathrm{p}_{1}, \mathrm{q}_{1} ; \mathrm{N}_{2}}^{0, \mathrm{n}_{1} ; \mathrm{N}_{1}}\{f(x, y) ; s, t\}
$$

\section{Special cases:}

\section{Case 1:}

If all the exponents $\xi_{j}\left(j=1, \ldots \ldots, p_{1}\right), \eta_{j}\left(j=1, \ldots \ldots, q_{1}\right), U_{j}\left(j=1, \ldots \ldots, p_{2}\right), V_{j}\left(j=1, \ldots \ldots, q_{2}\right)$, 
$P_{j}\left(j=1, \ldots \ldots, p_{3}\right), Q_{j}\left(j=1, \ldots \ldots, q_{3}\right)$ are equal to unity and $n_{1}=0$,then (2.1) will reduce to the

$\mathrm{H}$-function of two variables defined by Mittal and Gupta [4] is as follows:

$$
\begin{aligned}
& \phi[f ; s, t]=\int_{0}^{\infty} \int_{0}^{\infty} e^{\frac{-s x}{a}} e^{\frac{-t y}{b}} \mathrm{I}_{\mathrm{p}_{1}, \mathrm{q}_{1} ; p_{2}, q_{2}: p_{3}, q_{3}}^{0,0 ; m_{2}, n_{2}: m_{3}, n_{3}}\left[\begin{array}{c|c}
\alpha s x & \left(\left(\mathrm{a}_{j}: \alpha_{j}, \mathrm{~A}_{j} ; 1\right)\right) ;\left(\left(\mathrm{c}_{j}, \mathrm{C}_{j} ; 1\right)\right) ;\left(\left(\mathrm{e}_{j}, \mathrm{E}_{j} ; 1\right)\right) \\
\beta t y & \left(\left(\mathrm{~b}_{j}: \beta_{j}, \mathrm{~B}_{j} ; 1\right)\right) ;\left(\left(\mathrm{d}_{j}, \mathrm{D}_{j} ; 1\right)\right) ;\left(\left(\mathrm{f}_{j}, \mathrm{~F}_{j} ; 1\right)\right)
\end{array}\right] f(x, y) d x d y \\
& \phi[f ; s, t]=\int_{0}^{\infty} \int_{0}^{\infty} e^{\frac{-s x}{a}} e^{\frac{-t y}{b}} \mathrm{H}_{\mathrm{p}_{1}, \mathrm{q}_{1} ; p_{2}, q_{2}: p_{3}, q_{3}}^{0, m_{2}, n_{2}: m_{3}, n_{3}}\left[\begin{array}{c|c}
\alpha s x & \left(\begin{array}{c}
\left.\mathrm{a}_{j}: \alpha_{j}, \mathrm{~A}_{j}\right) ;\left(\mathrm{c}_{j}, \mathrm{C}_{j}\right) ;\left(\mathrm{e}_{j}, \mathrm{E}_{j}\right) \\
\beta t y
\end{array}\right. \\
\left(\mathrm{b}_{j}: \beta_{j}, \mathrm{~B}_{j}\right) ;\left(\mathrm{d}_{j}, \mathrm{D}_{j}\right) ;\left(\mathrm{f}_{j}, \mathrm{~F}_{j}\right)
\end{array}\right] f(x, y) d x d y,
\end{aligned}
$$

Provided the double integral transform on the right hand side of (2.2) exists and defines the double

$\mathrm{H}$-function transform of the given function $f(x, y)$ under the following conditions.

1. $0<a<\infty, 0<b<\infty, s, t \neq 0$

2. All the convergence conditions of H-function of two variables $x$ and $y$ defined in the region $D: 0 \leq x \leq \infty$, $0 \leq y \leq \infty$ such that the product $x^{\alpha} \cdot y^{\beta} \cdot f(x, y)$ is integrable over the finite region.

$D:\left(D_{1}, D_{2}\right): 0 \leq x \leq D_{1}, 0 \leq y \leq D_{2}, D_{1}>0, D_{2}>0$,

Where

$\alpha=\min _{0 \leq j \leq m_{2}}\left[\operatorname{Re}\left(\frac{d_{j}}{D_{j}}\right)\right], \quad \beta=\min _{0 \leq j \leq m_{3}}\left[\operatorname{Re}\left(\frac{f_{j}}{F_{j}}\right)\right]$,

Case 2:-

In (2.2), taking $a=\infty, b=\infty$, then we get new two dimensional H-function transform [8, eq.(3), p.93].

$$
\phi[f ; s, t]=\int_{0}^{\infty} \int_{0}^{\infty} \mathrm{H}_{\mathrm{p}_{1}, \mathrm{q}_{1} ; p_{2}, q_{2}: p_{3}, q_{3}}^{0,0 ; m_{3}, n_{3}: m_{3}, n_{3}}\left[\begin{array}{c|c}
\alpha s x & \left(\mathrm{a}_{j}: \alpha_{j}, \mathrm{~A}_{j}\right) ;\left(\mathrm{c}_{j}, \mathrm{C}_{j}\right) ;\left(\mathrm{e}_{j}, \mathrm{E}_{j}\right) \\
\beta\left(\mathrm{b}_{j}: \beta_{j}, \mathrm{~B}_{j}\right) ;\left(\mathrm{d}_{j}, \mathrm{D}_{j}\right) ;\left(\mathrm{f}_{j}, \mathrm{~F}_{j}\right)
\end{array}\right] f(x, y) d x d y,
$$

Provided

$$
\alpha=\min _{0 \leq j \leq m_{2}}\left[\operatorname{Re}\left(\frac{d_{j}}{D_{j}}\right)\right], \quad \beta=\min _{0 \leq j \leq m_{3}}\left[\operatorname{Re}\left(\frac{f_{j}}{F_{j}}\right)\right],
$$

\section{Case 3:-}

If we take $p_{1}=q_{1}=n_{1}=0$ in (2.1) then transform degenerates into product of two I-function of one variable introduced by Rathie A.K. [7] as,

$$
\phi[f ; s, t]=\int_{0}^{\infty} \int_{0}^{\infty} e^{\frac{-s x}{a}} e^{\frac{-t y}{b}} \mathrm{I}_{p_{2}, q_{2}}^{m_{2}, n_{2}}\left[\alpha s x \mid \begin{array}{l}
\left(\mathrm{c}_{j}, \mathrm{C}_{j} ; \mathrm{U}_{j}\right)_{1, p_{2}} \\
\left(\mathrm{~d}_{j}, \mathrm{D}_{j}: \mathrm{V}_{j}\right)_{1, q_{2}}
\end{array}\right] \times \mathrm{I}_{p_{3}, q_{3}}^{m_{3}, n_{3}}\left[\beta t y \mid \begin{array}{l}
\left(\mathrm{e}_{j}, \mathrm{E}_{j} ; \mathrm{P}_{j}\right)_{1, p_{3}} \\
\left(\mathrm{f}_{j}, \mathrm{~F}_{j}: \mathrm{Q}_{j}\right)_{1, q_{3}}
\end{array}\right] f(x, y) d x d y,
$$

Transform (2.5) is valid under condition obtainable and those with [8, Eq. (xvi), p. 90] hold.

Case 4:-

If $p_{1}=q_{1}=0$ in (2.2) then transform degenerates into product of two $\mathrm{H}$-function of one

Variable given by Singhe, Gir-Raj [9], as, 


$$
\begin{aligned}
& \phi[f ; s, t]=\int_{0}^{\infty} \int_{0}^{\infty} e^{\frac{-s x}{a}} e^{\frac{-t y}{b}} \mathrm{H}_{0,0 ; p_{2}, q_{2}: p_{3}, q_{3}}^{0,0 ; m_{2}, n_{2}: m_{3}, n_{3}}\left[\begin{array}{c}
\alpha s x \\
\beta t y
\end{array} \mid \begin{array}{l}
-,\left(\mathrm{c}_{j}, \mathrm{C}_{j}\right),\left(\mathrm{e}_{j}, \mathrm{E}_{j}\right) \\
-,\left(\mathrm{d}_{j}, \mathrm{D}_{j}\right),\left(\mathrm{f}_{j}, \mathrm{~F}_{j}\right)
\end{array}\right] f(x, y) d x d y \\
& \phi[f ; s, t]=\int_{0}^{\infty} \int_{0}^{\infty} e^{\frac{-s x}{a}} e^{\frac{-t y}{b}} \mathrm{H}_{p_{2}, q_{2}}^{m_{2}, n_{2}}\left[\alpha s x \mid \begin{array}{l}
\left(\left(\mathrm{c}_{j}, \mathrm{C}_{j}\right)\right) \\
\left.\left(\mathrm{(}_{j}, \mathrm{D}_{j}\right)\right)
\end{array}\right] \times \mathrm{H}_{p_{3}, q_{3}}^{m_{3}, n_{3}}\left[\beta t y \mid \begin{array}{l}
\left(\left(\mathrm{e}_{j}, \mathrm{E}_{j}\right)\right) \\
\left(\left(\mathrm{f}_{j}, \mathrm{~F}_{j}\right)\right)
\end{array}\right] f(x, y) d x d y,
\end{aligned}
$$

Provided

$$
\alpha=\min _{0 \leq j \leq m_{2}}\left[\operatorname{Re}\left(\frac{d_{j}}{D_{j}}\right)\right], \quad \beta=\min _{0 \leq j \leq m_{3}}\left[\operatorname{Re}\left(\frac{f_{j}}{F_{j}}\right)\right],
$$

Case 5:- In (2.1) putting

$\alpha_{j}=\mathrm{A}_{j}=\xi_{j}=C_{j}=U_{j}=E_{j}=P_{j}=\beta_{j}=B_{j}=\eta_{j}=D_{j}=V_{j}=F_{j}=Q_{j}=1$

And using the relation [3, p. 297 (45)], we get transform for G-function of two variables as,

$$
\begin{aligned}
& \phi[f ; s, t]=\int_{0}^{\infty} \int_{0}^{\infty} e^{\frac{-s x}{a}} e^{\frac{-t y}{b}} \mathrm{I}_{\mathrm{p}_{1}, \mathrm{q}_{1} ; p_{2}, q_{2}: p_{3}, q_{3}}^{0, \mathrm{n}_{1} ; m_{2}, n_{2}: m_{3}, n_{3}}\left[\begin{array}{c|c}
\alpha s x & \left(\mathrm{a}_{j}: 1,1 ; 1\right)_{1, p_{1}} ;\left(\mathrm{c}_{j}, 1 ; 1\right)_{1, p_{2}} ;\left(\mathrm{e}_{j}, 1 ; 1\right)_{1, p_{3}} \\
\beta t y & \left(\mathrm{~b}_{j}: 1,1 ; 1\right)_{1, q_{1}} ;\left(\mathrm{d}_{j}, 1 ; 1\right)_{1, q_{2}} ;\left(\mathrm{f}_{j}, 1 ; 1\right)_{1, q_{3}}
\end{array}\right] f(x, y) d x d y \\
& \phi[f ; s, t]=\int_{0}^{\infty} \int_{0}^{\infty} e^{\frac{-s x}{a}} e^{\frac{-t y}{b}} \mathrm{G}_{\mathrm{p}_{1}, \mathrm{q}_{1} ; p_{2}, q_{2}: p_{3}, q_{3}}^{0, \mathrm{n}_{1} ; m_{2}, n_{2}: m_{3}, n_{3}}\left[\begin{array}{c|c}
\alpha s x & \left(\mathrm{a}_{p_{1}}\right) ;\left(\mathrm{c}_{p_{2}}\right) ;\left(\mathrm{e}_{p_{3}}\right) \\
\beta t y & \left(\mathrm{~b}_{q_{1}}\right) ;\left(\mathrm{d}_{q_{2}}\right) ;\left(\mathrm{f}_{q_{3}}\right)
\end{array}\right] f(x, y) d x d y,
\end{aligned}
$$

The sets of conditions mentioned with (2.2) and [8, Eq. 12.3, p.7] will satisfy.

\section{References:-}

1. Braaksma, B.L.J. (1963), "Asymptotic expansions and analytic continuations for a class of Barnesintegrals." Compositio Math. 15, p. 339-341.

2. K. Shantha kumari, T. M. Vasudevan Nambisan, A. K. Rathie (2014), "A Study of I-Functions of Two Variables.” Le Matematiche Vol. LXIX (2014) -Fasc. I, p. 285-305.

3. Meijer C.S. (1964), "On the G-function I-VIII, Nederl. Akad. Wetensch. Proc. (49) 227-237, 344-356, $437-$ 469, 632-641, 765-772, 936-943, 1069-1072, 1165-1175.

4. Mittal P.K. and Gupta K.C. (1972), "An integral involving generalized function of two variables." Indian J. Pure and App. Math.5, pp.430-437.

5. Pragathi Y. Kumar, Hailu Gebreegziabher,Alen Mabrahtu, Satyanarayana B.(2015), "Derivative formulae involving I-function of two variables and generalized M-series." IARJSET (2) 6, pp. 95-98.

6. Rainvelly, E.D. (1963), "Special Functions" Macmillan Publisher (N.Y.)

7. Rathie, A.K. (1997), "A new generalization of generalized hypergeometric function, Le-Mathmatiche, 52 (2), pp. 297-310.

8. Shrivastava, H.M., K.C. Gupta and S.P. Goyal (1982), "The H-function of One and Two variables with applications." South Asian Publications, New Delhi.

9. Singhe, GirRaj (2013) "A study of I-function of two variables, thesis, Bark.Univ.(Bhopal).

10. Vyas, V.M., Rathie, A.K. (1998), “A study of I-function.” Vijanana Parishad Anusandhan Patrika, 41 (3), pp. 185-191.

11. Vyas, V.M., Rathie, A.K. (1998), "A study of I-function-II" Vijanana Parishad Anusandhan Patrika, 41 (4), pp. 253-257. 